\title{
Produção de sementes de soja enriquecidas com molibdênio ${ }^{1}$
}

\author{
Carina Oliveira e Oliveira², Carolina Cipriano Pinto ${ }^{3}$, Ariani Garcia ${ }^{2}$, João Victor Trombeta Bettiol', \\ Marco Eustáquio de Sá2, Edson Lazarini²,
}

10.1590/0034-737X201764030009

\section{RESUMO}

O objetivo deste trabalho foi avaliar a produção de sementes de soja, em função da aplicação de doses de molibdênio (Mo) foliar, e o crescimento e produção das plantas advindas das sementes obtidas desse cultivo. O cultivar utilizado foi o BRS Valiosa RR, no primeiro ano, sendo utilizadas três doses de Mo (0, 400 e $\left.800 \mathrm{~g} \mathrm{ha}^{-1}\right)$, com quatro repetições. Com as sementes colhidas formaram-se três lotes de sementes, com diferentes quantidades de Mo (9,27; 51,56 e 65,43 $\mathrm{mg} \mathrm{kg}^{-1} \mathrm{de} \mathrm{Mo}$ ). No segundo ano, utilizando-se as sementes anteriormente produzidas, montaram-se dois experimentos, conduzidos paralelamente. O primeiro foi realizado em blocos casualizados, em esquema fatorial 3 x 3: três métodos de aplicação de Mo (sem aplicação, tratamento de sementes e por via foliar, no estádio $\mathrm{V}_{4}$, dose de $25 \mathrm{~g} \mathrm{ha}^{-1}$ de Mo) e os três lotes de sementes enriquecidas do ano anterior, com quatro repetições. O segundo foi realizado em blocos casualizados, em esquema fatorial 4 x 4 x 3 [épocas de aplicação $\left(V_{4}\right.$, no pré- florescimento, $R_{3}$ e $\left.R_{5}\right)$ x doses de Mo foliar $\left(0,25,50\right.$ e $\left.100 \mathrm{~g} \mathrm{ha}^{-1}\right)$ x lotes de sementes enriquecidas com Mo do ano anterior], com quatro repetições. Conclui-se que o teor de proteína das sementes é $9 \%$ maior com a presença de Mo, independentemente do modo como é fornecido. A utilização de Mo influi positivamente na produtividade de soja, quando fornecido pelo uso de sementes enriquecidas com teor de até $51,56 \mathrm{mg} \mathrm{kg}^{-1}$, ou aplicação por via foliar, com doses entre 25 e $50 \mathrm{~g} \mathrm{ha}^{-1}$.

Palavras-chave: Glycine $\max (\mathrm{L}$.$) Merrill; micronutriente; qualidade nutricional.$

\section{ABSTRACT}

\section{Production of soybean seeds enriched with molybdenum}

The objective was to evaluate the production of seeds enriched by the application of foliar molybdenum (Mo) doses and the growth and yield of plants resulting from the seeds obtained in this cultivation. We used the cultivar BRS Valiosa RR and in the first year, we used three doses of molybdenum $\left(0,400\right.$, and $\left.800 \mathrm{~g} \mathrm{ha}^{-1}\right)$, with four replications. With the harvested seeds, three lots of seeds with different Mo contents were formed $\left(9.27 ; 51.56\right.$, and $65.43 \mathrm{mg} \mathrm{kg}^{-1}$ of Mo). In the second year, using the seeds produced, two experiments were set, conducted in parallel. The first experiment was arranged in a randomized block design in a $3 \times 3$ scheme with four replications - three application methods (without application, at sowing, and foliar in the $\mathrm{V}_{4}$ stage at the dose of $25 \mathrm{~g} \mathrm{ha}^{-1} \mathrm{Mo}$ ) and the three lots of seeds from the previous year. The second experiment was arranged in a randomized block design in a $4 \times 4 \times 3$ scheme with four replications - four application stages $\left(\mathrm{V}_{4}\right.$, in pre-flowering, in $\mathrm{R}_{3}$, and $\left.\mathrm{R}_{5}\right) \times$ four foliar Mo doses $(0,25,50$, and $100 \mathrm{~g}$ ha' $\left.{ }^{1}\right) \times$ three batches of seeds enriched with different Mo contents from the previous year. The protein content in seeds was $9 \%$ higher in the presence of Mo, regardless of how this was provided. The use of Mo is positive for soybean yield, when provided by the use of seeds enriched with a level of up to $51.56 \mathrm{mg} \mathrm{kg}^{-1}$ or by foliar application at doses between 25 and $50 \mathrm{~g} \mathrm{ha}^{-1}$.

Key words: Glycine $\max ($ L.) Merrill; micronutrient; nutritional quality.

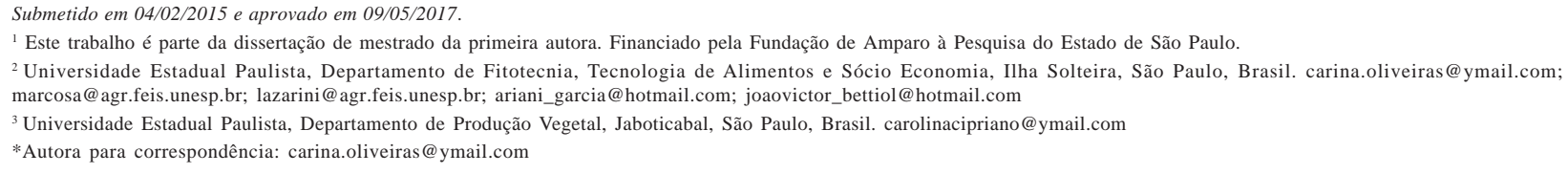




\section{INTRODUÇÃO}

O molibdênio (Mo) é um elemento que compõe pelo menos cinco enzimas distintas, que catalizam reações muito diversas (nitrogenase, redutase do nitrato, oxidase de aldeído, oxidase do sulfato e oxidase da xantina), participando de processos de transferência elétrica (Malavolta, 2006), sendo também requerido na fixação biológica de nitrogênio (FBN). No processo da FBN, o Mo atua na redutase do nitrito, especificamente na transferência de elétrons para o nitrito; além disto, o Mo, na forma de FeMo proteína, faz parte da reação de quebra da ligação tripla do $\mathrm{N}$ atmosférico, resultando em $\mathrm{NH}_{3}+\mathrm{H}_{2}$, sendo deste modo assimilado pela planta.

O Mo pode ser aplicado de quatro modos: (i) diretamente no solo, junto com a adubação convencional (Cheng, 1985), sendo, porém, assim como o fósforo, adsorvido pelos óxidos de ferro ( $\mathrm{Fe}$ ) e alumínio (Al), principalmente em solos muito intemperizados (Santos, 2012); (ii) por via de tratamento de sementes (Parducci et al., 1989); entretanto, estudos como o de Campo et al. (1999) verificaram que a aplicação de Mo na forma salina afetou a sobrevivência da bactéria; (iii) por aplicação foliar que, segundo Pessoa (1998), produz efeitos nas características da planta; (iv) por uso de sementes enriquecidas que, segundo a Embrapa (2003), é um método alternativo que apresenta resultados positivos no aumento da fixação biológica de nitrogênio e da produtividade da soja.

De acordo Lima (2009), se a pulverização foliar for realizada no final do estádio de desenvolvimento das sementes, grande quantidade de Mo é transferida para elas, pois os nutrientes estão sendo direcionados para sua formação, obtendo-se sementes enriquecidas desse elemento. Este método de utilizar Mo presente na reserva da semente traz a vantagem de que o nutriente estará disponível de acordo com a demanda da planta, aumentando, assim, a sua eficiência, sendo de baixo custo e tecnicamente viável, desde que na concentração adequada do elemento, que é de 22,4 $\mathrm{mg} \mathrm{kg}^{-1}$ (Harris et al.1965).

Considerando-se que solos anteriormente sob vegetação de cerrado podem apresentar limitações quanto ao conteúdo de molibdênio, este trabalho teve por objetivo estudar a produção de sementes enriquecidas, em função da aplicação de doses de Mo foliar, e o desempenho de plantas advindas de sementes enriquecidas.

\section{MATERIAL E MÉTODOS}

\section{Área experimental}

Os experimentos foram realizados na área experimental da Fazenda de Ensino, Pesquisa e Extensão da Faculdade de Engenharia da UNESP - Campus de Ilha Solteira, no município de Selvíria, Estado do Mato Grosso do Sul (MS), nas coordenadas geográficas $51^{\circ} 22^{\prime} \mathrm{O}, 20^{\circ} 22^{\prime} \mathrm{S}$, em
$335 \mathrm{~m}$ de altitude. Os cultivos foram implantados em Latossolo Vermelho distrófico (LVd) com textura argilosa. Os dados climáticos durante a condução dos experimentos estão apresentados na Figura 1, indicando com as setas os dias de aplicação, em ambos os anos.

Foi realizada análise de solo nos dois anos de experimento:

Primeiro ano: $\mathrm{pHem} \mathrm{CaCl}_{2} 0,01 \mathrm{~mol} \mathrm{~L}^{1}=4,9 ;$ M.O. $=31 \mathrm{~g}$ $\mathrm{kg}^{1} ; \mathrm{P}=42 \mathrm{mg} \mathrm{dm}^{3} ;$ e em mmol $\mathrm{dm}^{3}: \mathrm{K}=3,8 ; \mathrm{Ca}=20 ; \mathrm{Mg}=$ $12 ; \mathrm{Al}=2 ; \mathrm{H}+\mathrm{Al}=38 ; \mathrm{SB}=35,8 ; \mathrm{CTC}=73,8 \mathrm{e} \mathrm{V}=49 \%$.

Segundo ano: $\mathrm{pH} \mathrm{em} \mathrm{CaCl}_{2} 0,01 \mathrm{~mol} \mathrm{~L}^{1}=5,0$; M.O.=

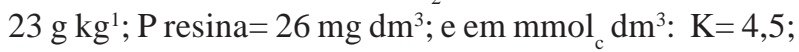
$\mathrm{Ca}=14 ; \mathrm{Mg}=8 ; \mathrm{Al}=2 ; \mathrm{H}+\mathrm{Al}=36 ; \mathrm{SB}=26,5 ; \mathrm{CTC}=62,5 \mathrm{e}$ $\mathrm{V}=42 \%$.

A semeadura da soja no primeiro ano ocorreu no dia 07/11/2012, em área com dez anos sob sistema plantio direto, em que a cultura antecessora foi o milho. Para preparo da área, foi realizada a dessecação e, posteriormente, o uso de desintegrador mecânico para manejo dos restos culturais. A adubação básica no sulco de semeadura, de acordo com as características químicas do solo, foi de 250 $\mathrm{kg} \mathrm{ha}^{-1}$ da formulação 08-28-16, objetivando alcançar produtividade de $3.400 \mathrm{~kg} \mathrm{ha}^{-1}$ de sementes. As sementes foram previamente tratadas com fungicida à base de carboxin + thiram, na concentração de $250 \mathrm{~mL}$ do p.c. 100 $\mathrm{kg}^{-1}$ de sementes, e inoculadas com inoculante líquido de Bradyrhizobium japonicum, usando-se $2 \mathrm{~mL}$ por $\mathrm{kg}$ de semente das estirpes SEMIA 5079 e SEMIA 5080, à base de $5 \times 10^{5}$ células viáveis $\mathrm{mL}^{-1}$. A densidade de semeadura foi de 16 sementes por metro de sulco, espaçadas de 0,50 $\mathrm{m}$ entre linhas, sendo as parcelas constituídas por sete linhas com 20,0 metros de comprimento.

Foram utilizados o cultivar BRS Valiosa RR, categoria $\mathrm{C} 2$, e três doses de molibdênio, 0,400 e $800 \mathrm{~g} \mathrm{ha}^{-1}$, usando-se produto com $15 \%$ de molibdênio e densidade de $1,32 \mathrm{~g} \mathrm{~L}^{-1}$, com quatro repetições. A aplicação foi realizada em duas épocas, metade da dose no estádio $R_{3}$ e a outra metade no estádio $R_{6}$, sendo os estádios descritos por Fehr et al. (1971). Para escolha das épocas de aplicação, foi tomado como base o trabalho de Campo \& Hungria (2003), que concluíram que duas aplicações de $400 \mathrm{~g}$ de Mo, entre os estádios $\mathrm{R}_{3}$ e $\mathrm{R}_{5.5}$, com intervalos de dez dias, são melhores que somente uma aplicação, para produção de sementes enriquecidas. As aplicações foram realizadas com pulverizador de barras acoplado a trator, calibrado para aplicação do volume de calda recomendado pelo fabricante do produto. $\mathrm{O}$ pulverizador estava equipado com pontas do tipo jato em leque, espaçadas de $0,50 \mathrm{~m}$.

A colheita foi realizada em 15/03/2013, manualmente, coletando-se as plantas das três linhas centrais, desprezando-se 2,0 $\mathrm{m}$ em cada extremidade. As sementes foram secadas até $13 \%$ de umidade e acondicionadas, em sacos de papel kraft, em câmara seca, a $20^{\circ} \mathrm{C}$ e $50 \%$ UR. 
As sementes colhidas no ano agrícola 2012-2013 foram utilizadas para dois experimentos do ano 2013-2014, sendo que os teores de Mo dos três lotes dessas sementes foram os seguintes: i) sem a aplicação de Mo, as sementes apresentam 9,27 $\mathrm{mg} \mathrm{kg}^{-1}$; ii) com a dose de $400 \mathrm{~g}$ $\mathrm{ha}^{-1} \mathrm{de}$ Mo, as sementes continham 51,56 $\mathrm{mg} \mathrm{kg}^{-1}$; iii) com a dose de $800 \mathrm{~g} \mathrm{ha}^{-1}$ de Mo, o teor nas sementes foi de $65,43 \mathrm{mg} \mathrm{kg}^{-1}$.

O experimento do ano agrícola 2012-2013 foi realizado com o intuito principal de produzir sementes com diferentes teores de Mo, mas foram feitas também avaliações das características agronômicas da produtividade e do teor de proteína, para se avaliar o comportamento da cultura e verificar se ocorreu alguma interferência que pudesse resultar em diferenças no experimento do ano posterior.

O Experimento 1 do ano 2013-2014 foi realizado em blocos casualizados, em esquema fatorial $3 \times 3$, sendo três métodos de aplicação de Mo (sem aplicação de molibdênio, no tratamento de sementes e, por via foliar, no estádio $\mathrm{V}_{4}$, sendo os dois últimos com a dose de $25 \mathrm{~g} \mathrm{ha}^{-1}$ de Mo) e os três lotes de sementes enriquecidas do ano 2012-2013, com quatro repetições. Utilizaram-se as sementes obtidas do cultivar BRS Valiosa RR no ano agrícola 2012-2013. O tratamento com o uso de Mo na semeadura foi realizado dia 29/11/2013 em V 4 , no dia 28/12/2013.
O Experimento 2 do ano 2013-2014 foi realizado em blocos casualizados, em esquema fatorial 4 x 4 x 3 (épocas de aplicação x doses de Mo foliar x lotes de sementes enriquecidas com Mo do ano anterior), com quatro repetições. As épocas de aplicação foram: $V_{4}$, no préflorescimento, em $\mathrm{R}_{3}$ e $\mathrm{R}_{5}$ (Fehr et al., 1971); as doses foliares utilizadas foram 0, 25, 50 e $100 \mathrm{~g} \mathrm{ha}^{-1} \mathrm{de} \mathrm{Mo,} \mathrm{nos}$ três lotes de sementes advindas de 2012-2013, do cultivar BRS Valiosa RR.

A aplicação do molibdênio foi realizada com pulverizador costal, sendo o molibdato de amônio, com $81 \%$ de Mo, aplicado conforme os tratamentos. As parcelas foram constituídas por sete linhas espaçadas de $0,45 \mathrm{~m}$ e com 5,0m de comprimento. Como área útil, foram consideradas as duas linhas centrais, desprezando-se $0,5 \mathrm{~m} \mathrm{em}$ cada extremidade.

O experimento 1 foi semeado no dia 29/11/2013 e, o experimento 2, em 30/11/2013, sendo que a área utilizada para ambos foi cultivada com feijão de inverno anteriormente. O preparo da área foi de uma aração, seguida de uma gradagem. A adubação no sulco de semeadura, de acordo com as características químicas do solo, foi de 250 $\mathrm{kg} \mathrm{ha}^{-1}$ da formulação 00-20-20. As sementes foram previamente tratadas com fungicida a base de carboxin + thiram, na concentração de $250 \mathrm{~mL}$ do p.c. $100 \mathrm{~kg}^{-1}$ de sementes,
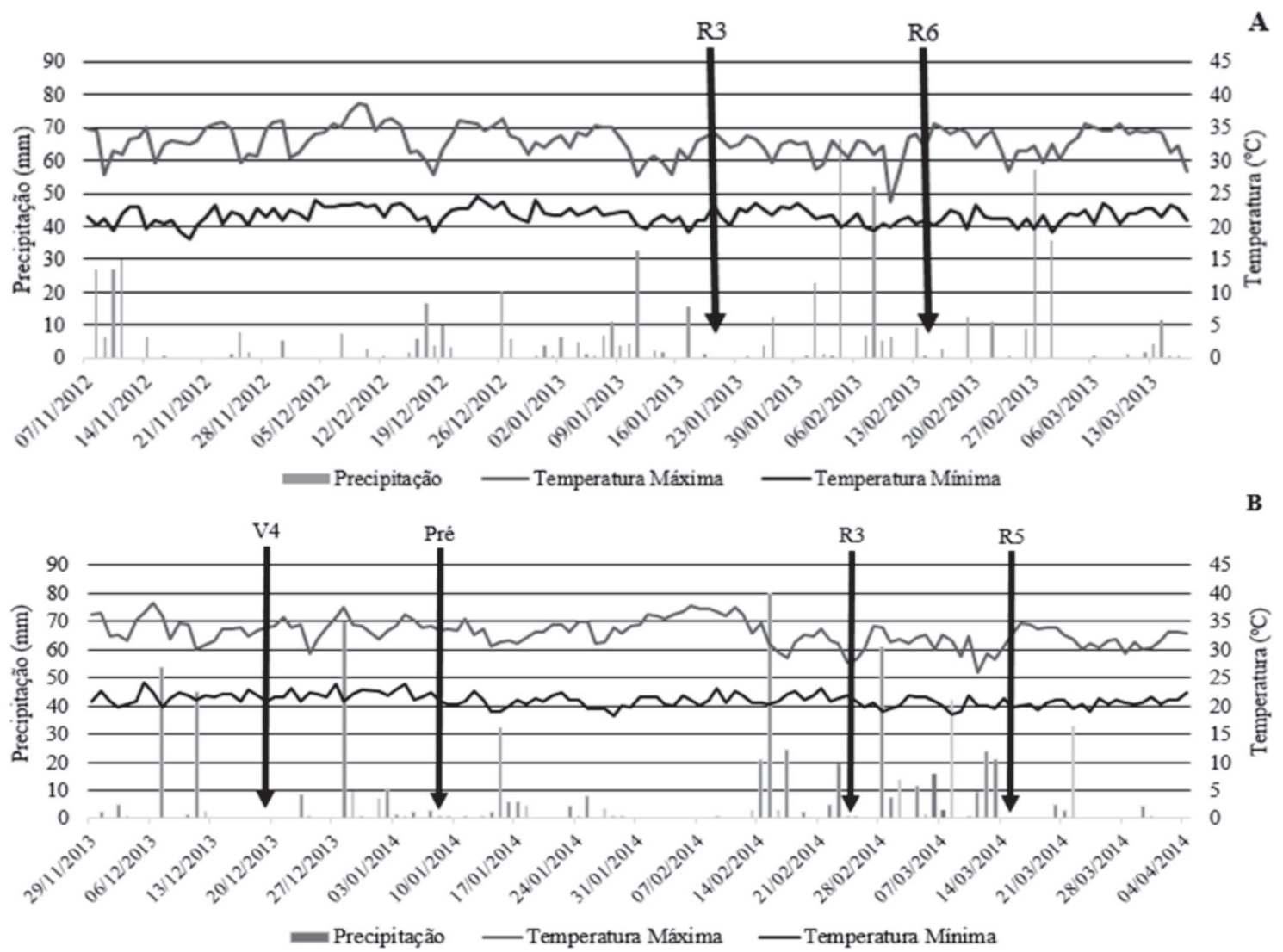

Figura 1: A. Valores médios de temperaturas máximas e mínimas e de precipitação pluvial no período de 07/11/2012 a 13/03/2013, em Selvíria, MS, 2013. B. Valores médios de temperaturas máximas e mínimas e precipitação pluvial no período de 29/11/2013 a 04/ $04 / 2014$.

Rev. Ceres, Viçosa, v. 64, n.3, p. 282-290, mai/jun, 2017 
inoculadas do mesmo modo, com o mesmo inoculante do ano anterior, e semeadas manualmente, visando à densidade de 16 plantas por metro de sulco. A colheita para ambos os experimentos ocorreu no dia 03/04/2014, realizada manualmente, colhendo-se todas as plantas da área útil de cada parcela.

Em ambos os anos, foi realizado o controle fitossanitário, com aplicação, no estádio $\mathrm{V}_{4}$ de imazethapyr (40 g i.a. ha $\left.{ }^{-1}\right)+$ lactofen $\left(72\right.$ g i.a. ha- $\left.{ }^{-1}\right)+$ chlorimuron-ethyl (15 g i.a. ha-1); no estádio $\mathrm{V}_{6}$ aplicação de $1,5 \mathrm{~L} \mathrm{ha}^{-1} \mathrm{de}$ glifosato e imidacloprid + betacyflutrina ( 90 g i.a. ha-1) + cipermetrina $0,2 \mathrm{~L} \mathrm{ha}^{-1}+$ piraclostrobina + epoxiconazol (105gi.a.ha $\left.{ }^{-1}\right)$; na pré-floração, aplicação de endosulfan (700 g i.a. ha-1) e clorantraniliprole (10 g i.a. ha $\left.{ }^{1}\right)+$ azoxistrobina + ciproconazol $(60+24$ g i.a. ha-1 $)$; no estádio $\mathrm{R}_{1}$ cipermetrina $\left(50 \mathrm{~g}\right.$ i.a. $\left.\mathrm{ha}^{-1}\right)+$ paration metílico (600 g i.a. ha ${ }^{-1}$ ); no estádio $\mathrm{R}_{3}$ aplicação de cipermetrina + pyraclostrobin + epoxiconazol $\left(66,5+25,0\right.$ g i.a. ha $\left.{ }^{-1}\right)$; no estádio $R_{5}$, aplicação de imidacloprido + betaciflutrina $\left(75+9,38 \mathrm{~g}\right.$ i.a. ha $\left.{ }^{-1}\right)+$ paration metílico $(600 \mathrm{~g}$ i.a. ha $\left.{ }^{-1}\right)+$ ciproconazol $\left(50\right.$ g i.a. ha $\left.{ }^{-1}\right)$; no estádio $R_{6}$, aplicação de cipermetrina $\left(50 \mathrm{~g}\right.$ i.a. $\left.\mathrm{ha}^{-1}\right)+$ paration metílico (600 g i.a. ha-1).

\section{Avaliações para ambos os anos}

Estande final: foi determinado pela contagem do número de plantas, em 3,0 m lineares, em uma das linhas da área útil de cada parcela, quando as plantas atingiram o estádio $\mathrm{R}_{8}$, segundo Fehr et al. (1971), ou seja, no momento de colheita das plantas para avaliação das características agronômicas e da produtividade de sementes.

Características agronômicas: quando as plantas nas parcelas experimentais atingiram o estádio $R_{8}$, foram coletadas, em uma das linhas da área útil da parcela, dez plantas, para determinação de:

- Altura de planta: distância entre o colo da planta e o ápice da haste principal.

- Número de vagens por planta: contagem de todas as vagens que apresentavam sementes, dividindo-se pelo número de plantas avaliadas.

- Número de sementes por vagem: número de sementes por planta dividido pelo número de vagens por planta.

Massa de 1000 sementes: pesagem em balança de precisão $(0,01 \mathrm{~g})$ de uma amostra de 1000 sementes de cada parcela. Após a pesagem, determinou-se a umidade das sementes pelo método da estufa $\left(105 \pm 3{ }^{\circ} \mathrm{C}\right)$, para correção dos valores a 13\% (base úmida) (Brasil, 2009).

Produtividade de sementes: na área útil da parcela, foram coletadas todas as plantas de duas linhas, com 4,0 $\mathrm{m}$ de comprimento. Essas plantas, após identificação e secagem a sol, foram trilhadas mecanicamente, as semen- tes obtidas foram pesadas e determinada, em aparelho digital, a umidade, para cálculo da produtividade de sementes e extrapolação para um hectare.

Teor de Proteína: secagem de uma amostra das sementes, em estufa de $105 \pm 3{ }^{\circ} \mathrm{C}$. e moagem em moinho tipo Willey, armazenagem do material moído em saquinhos plásticos colocados em câmara seca. A metodologia para determinação do nitrogênio é descrita por Malavolta et al. (1997), que consiste na transformação do $\mathrm{N}$ amoniacal em amônia, a qual é fixada pelo ácido bórico e, posteriormente, titulada com $\mathrm{H}_{2} \mathrm{SO}_{4}$ até nova formação de $\mathrm{N}$ amoniacal na presença de indicador ácido-base, multiplicado pelo fator 6,25 (AOSA., 1983).

\section{Análise estatística}

Os dados foram submetidos ao teste $\mathrm{F}$ da análise de variância e, quando constatada diferenças entre as médias dos tratamentos, foi realizado o teste de Tukey a $5 \%$ de probabilidade $(\mathrm{p}<0,05)$. Para avaliação das doses de molibdênio, foi realizada regressão polinomial, ajustando-se modelos de equações significativas pelo teste $\mathrm{F}$ ( $\mathrm{p}$ $<0,01$ e $\mathrm{p}<0,05)$. O aplicativo computacional utilizado foi o SISVAR (Ferreira, 2010).

\section{RESULTADOS E DISCUSSÃO}

\section{Enriquecimento das sementes com Mo (2012-} 2013)

Observa-se que as características agronômicas, assim como os demais parâmetros avaliados, não foram influenciados significativamente pelas doses de Mo (Tabela 1). Nakao et al. (2014), testando quatro doses de Mo foliar com o mesmo cultivar, verificaram médias maiores para altura de plantas, número de vagens por planta e número de sementes por vagem, mas, assim como neste trabalho, não se observaram efeito significativo das doses de Mo. A produtividade média de $3.692 \mathrm{~kg} \mathrm{ha}^{-1}$, na safra 20122013 (Tabela 1), foi superior à média brasileira que, segundo os dados da Conab (2014), foi de $2.938 \mathrm{~kg} \mathrm{ha}^{-1}$. Possenti \& Villela (2010), estudando a produção de sementes enriquecidas com Mo, não verificaram diferença significativa para produtividade, semelhantemente ao encontrado neste trabalho.

A aplicação de Mo foliar ocorreu nos estádios reprodutivos da soja (metade em $\mathrm{R}_{3}$ e metade em $\mathrm{R}_{5}$ ). Consequentemente, as plantas já haviam definido características como a altura e estavam definindo as demais características; porém, o tempo para atuação do Mo foi curto, pois a soja entra em maturidade fisiológica em $R_{7}$. Assim sendo, é justificável que algumas características, como altura de plantas, vagens por planta, sementes por vagem, estande final e massa de 1000 sementes não sejam alteradas em função das aplicações do produto. 
Os dados para teor de proteína das sementes ajustaram-se à equação linear crescente (Tabela 1), mostrando que, conforme se aumentaram as doses de Mo, aumentaram-se também os teores de proteínas das sementes. Isto pode ser explicado pelo fato de que o Mo faz parte da molécula da nitrogenase e da enzima redutase nitrato, que estão diretamente ligadas ao metabolismo do $\mathrm{N}$ das plantas de soja, o que pode influenciar na maior incorporação de N.

\section{Uso de sementes enriquecidas: Experimento 1 - safra 2013-2014}

A altura de planta, as características de produção e o estande não foram influenciados nem pela utilização de sementes enriquecidas com Mo, nem pelos métodos de aplicação de Mo. Já a massa de 1000 sementes e a produtividade foram influenciados, significativamente, pelos métodos de aplicação (Tabela 2). Este resultado é similar ao verificado por Possenti et al. (2009) que utilizaram se-

Tabela 1: Valores de F e médias de altura de plantas (AP), número de vagens por planta (VP), número de sementes por vagem (SV), estande (E), massa de 1000 sementes (MM), produtividade (PROD) e percentagem de proteína (PB) em sementes de soja, em função de doses de molibdênio foliar

\begin{tabular}{|c|c|c|c|c|c|c|c|}
\hline \multirow{2}{*}{ Tratamentos } & $\mathbf{A P}$ & VP & SV & $\mathbf{E}$ & MM & PROD & PB \\
\hline & cm & \multicolumn{2}{|c|}{$\mathbf{n}^{\mathbf{0}}$} & plantas ha ha $^{-1}$ & $\mathbf{g}$ & kg ha $^{-1}$ & $\%$ \\
\hline \multicolumn{8}{|c|}{ Doses Mo (D) $\left(\mathrm{g} \mathrm{ha}^{-1}\right)$} \\
\hline 0 & 87,48 & 58,25 & 1,90 & 232.531 & 162,0 & 3.804 & 41,01 \\
\hline 400 & 99,17 & 54,00 & 1,91 & 232.612 & 163,6 & 3.730 & 43,14 \\
\hline 800 & 97,13 & 50,00 & 1,89 & 231.569 & 164,8 & 3.542 & 43,48 \\
\hline
\end{tabular}

Teste $\mathrm{F}$

\begin{tabular}{lccccccc} 
(D) & $1,84^{\mathrm{NS}}$ & $2,61^{\mathrm{NS}}$ & $0,05^{\mathrm{NS}}$ & $3,77^{\mathrm{NS}}$ & $0,93^{\mathrm{NS}}$ & $0,74^{\mathrm{NS}}$ & $3,80^{\mathrm{NS}}$ \\
\hline C.V. $(\%)$ & 10,16 & 15,26 & 5,60 & 10,27 & 2,45 & 8,51 & 3,22 \\
\hline Média Geral & 94,86 & 51,75 & 1,90 & 232.204 & 163,6 & 3.692 & 42,54
\end{tabular}

Médias seguidas de mesma letra, na coluna, não diferem entre si pelo teste de Tukey a $5 \%$ de probabilidade.

NS $=$ não significativo; $*$ significativo a $5 \%$ de probabilidade; $* *$ = significativo a $1 \%$ de probabilidade.

$\mathrm{y}$ (proteína) $=0,003081 \mathrm{x}+41,310 \mathrm{R}^{2}=0,85$

Tabela 2: Valores de F e médias de altura de plantas (AP), número de vagens por planta (VP), número de sementes por vagem (SV), estande (E), massa de 1000 sementes (MM), produtividade (PROD) e percentagem de proteína (PB), de sementes de soja, em função de métodos de aplicação de molibdênio e da utilização de sementes enriquecidas com diferentes doses de Mo

\begin{tabular}{|c|c|c|c|c|c|c|c|}
\hline \multirow[b]{2}{*}{ Tratamentos } & $\mathbf{A P}$ & $\mathbf{V P}^{(1)}$ & SV & \multirow{2}{*}{$\frac{E}{\text { plantas ha }^{-1}}$} & \multirow{2}{*}{$\begin{array}{c}\text { MM } \\
\mathrm{g}\end{array}$} & \multirow{2}{*}{$\begin{array}{c}\text { PROD } \\
\text { kg ha }^{-1}\end{array}$} & \multirow{2}{*}{$\begin{array}{c}\text { PB } \\
\%\end{array}$} \\
\hline & $\mathbf{c m}$ & \multicolumn{2}{|c|}{$\mathbf{n}^{\mathbf{0}}$} & & & & \\
\hline \multicolumn{8}{|c|}{ Métodos de Aplicação (M) } \\
\hline Sem Aplicação & 68,2 & 74,4 & 2,11 & 242.350 & $145,1 \mathrm{~b}$ & $5.474 \mathrm{ab}$ & 41,35 \\
\hline Tratamento de Sementes & 73,2 & 64,6 & 2,06 & 250.000 & $162,2 \mathrm{a}$ & $4.458 \mathrm{~b}$ & 42,01 \\
\hline Via Foliar & 78,5 & 76,7 & 2,15 & 236.800 & $149,7 \mathrm{~b}$ & $6.036 \mathrm{a}$ & 41,16 \\
\hline \multicolumn{8}{|c|}{ Sementes enriquecidas com doses de Mo (S) } \\
\hline \multicolumn{8}{|l|}{$\left(\mathrm{g} \mathrm{ha}^{-1}\right)$} \\
\hline$\overline{0}$ & 74,0 & 64,4 & 2,13 & 240.500 & 150,7 & 5.269 & 41,14 \\
\hline 400 & 74,3 & 79,3 & 2,09 & 242.350 & 152,0 & 5.556 & 41,74 \\
\hline 800 & 71,7 & 62,0 & 2,10 & 246.300 & 154,3 & 5.142 & 41,63 \\
\hline \multicolumn{8}{|l|}{ Teste F } \\
\hline$(\mathrm{M})$ & $2,44^{\mathrm{NS}}$ & $4,68^{\mathrm{NS}}$ & $0,78^{\mathrm{NS}}$ & $0,18^{\mathrm{NS}}$ & $22,39^{* *}$ & $3,73^{*}$ & $1,21^{\mathrm{NS}}$ \\
\hline (S) & $0,19^{\mathrm{NS}}$ & $2,51^{\mathrm{NS}}$ & $0,20^{\mathrm{NS}}$ & $0,02^{\mathrm{NS}}$ & $0,97^{\mathrm{NS}}$ & $0,26^{\mathrm{NS}}$ & $0,62^{\mathrm{NS}}$ \\
\hline$(\mathrm{M}) \mathrm{x}(\mathrm{S})$ & $0,36^{\mathrm{NS}}$ & $0,57^{\mathrm{NS}}$ & $0,60^{\mathrm{NS}}$ & $1,71^{\mathrm{NS}}$ & $0,22^{\mathrm{NS}}$ & $0,88^{\mathrm{NS}}$ & $4,28^{*}$ \\
\hline DMS (5\%) & 11,90 & 21,09 & 0,18 & 57297 & 6,40 & 1.451 & 1,47 \\
\hline C.V. $(\%)$ & 13,48 & 12,54 & 7,00 & 11,80 & 6,02 & 26,93 & 2,94 \\
\hline Média Geral & 73,3 & 68,6 & 2,11 & 243.050 & 152,3 & 5.322 & 41,50 \\
\hline
\end{tabular}

(1) Dados transformados em $(\mathrm{X}+0,5)^{\wedge} 0,5$

Médias seguidas de mesma letra, na coluna, não diferem entre si pelo teste de Tukey a $5 \%$ de probabilidade.

Ns $=$ não significativo; $*$ = significativo a $5 \%$ de probabilidade; $* *=$ significativo a $1 \%$ de probabilidade.

Rev. Ceres, Viçosa, v. 64, n.3, p. 282-290, mai/jun, 2017 
mentes enriquecidas e não verificaram efeitos sobre os componentes de rendimento avaliados.

Para produtividade, verificou-se diferença entre os métodos de aplicação e, como se observa, o efeito da aplicação no tratamento de sementes diferiu do efeito da aplicação foliar em $\mathrm{V}_{4}$, com $1.578 \mathrm{~kg} \mathrm{ha}^{-1}$ a menos (Tabela 2). Isto pode ter ocorrido por toxicidade prejudicial à bactéria (Bradyrhizobium japonicum), causada pelo adubo molibdíco, com reflexos diretos na produtividade. Esta toxicidade já foi verificada por Albino \& Campo (2001), que relatam menor número de células de Bradyrhizobium ao aplicarem o Mo às sementes antes do inoculante.

Analisando-se a massa de 1000 sementes (Tabela 2), observa-se que a utilização de $25 \mathrm{~g}$ de Mo junto com o tratamento de sementes apresentou diferença com as massas obtidas com os demais métodos, com as sementes apresentando maior massa. Ao se verificar a relação entre alguns dados obtidos, como o número de sementes por vagem e massa de 1000 sementes, observa-se que, com o tratamento de sementes alcançou-se menor número de sementes por vagem, com o que se tem mais espaço para o crescimento de cada grão, maior acúmulo de massa e menor competição, além de a cultura apresentar o efeito compensatório de crescimento de sementes. Por esse efeito compensatório em plantas de soja, foi verificado que mesmo o estande abaixo do desejado pouco ou nenhum efeito tem sobre a produtividade de grãos.

Quanto ao teor de proteína das sementes, ocorreu interação significativa entre métodos de aplicação e utilização de sementes enriquecidas com doses de Mo (Tabela 3). A percentagem de proteína diferiu, entre sementes advindas de plantas que se originaram de sementes não enriquecidas com Mo, em relação aos métodos de aplicação de Mo. Com o uso de Mo no tratamento de sementes, obteve-se teor de proteína maior que o das sementes que não receberam Mo. Quanto aos métodos de aplicação, quando não se utilizou aplicação de Mo, observou-se ajuste linear crescente, mostrando que, quanto maior o teor de Mo no tratamento de sementes, maior a percentagem de proteína das sementes advindas dessas plantas. Isto indica a importância do Mo no metabolismo do N, em que o Mo desempenha um papel importante, participando da nitrogenase, que é responsável pela fixação de nitrogênio no nódulo, que, para a soja, é essencial, pois este processo supre sua necessidade de nitrogênio (Mengel \& Kirkby, 2001).

Meschede et al. (2004), estudando sementes com e sem tratamento com cobalto (Co) e Mo, verificaram que o uso desses micronutrientes aumentou o teor de proteína, assim como observado neste trabalho. Em contrapartida, Moraes et al. (2008), avaliando o efeito de Co e Mo, em diferentes concentrações e estádios de desenvolvimento da cultura, observaram que os teores de proteína em grãos de soja não apresentaram diferenças. As respostas das plantas ao Co e Mo são variáveis, pois o molibdênio é adsorvido pelos óxidos hidróxidos de $\mathrm{Fe}$ e Al, o que ocorre por causa dos processos de intemperismo nos solos tropicais (Malavolta et al., 1997). Assim, o íon $\mathrm{OH}^{-}$deslocaria o molibdato adsorvido nos coloides do solo, liberando-o na solução, enquanto os óxidos de $\mathrm{Al}$ e $\mathrm{Fe}$ o adsorveriam fortemente. Assim sendo, o fornecimento de Mo em épocas diferentes ou em diferentes doses pode dar respostas distintas à aplicação desse micronutriente.

\section{Uso de sementes enriquecidas: Experimento 2 - safra 2013-2014}

Nas análises para vagens por planta e massa de 1000 sementes, não se observou influência dos tratamentos (Tabela 4). Já, para a altura de plantas e sementes por vagem, houve diferença para doses foliares de Mo. Trabalhos como de $\mathrm{Oz}$ (2008) e de Souza et al. (2010) constataram o efeito compensatório de plantas de soja, ou seja, com menor número de plantas ocorre um aumento do número de ramificações, de vagens por planta e da massa de mil grãos, havendo, portanto, uma compensação da produtividade final.

Esses autores ainda observaram que em área com maior número de plantas pode ocorrer competição intraespecífica, acarretando menor ramificação e maior altura de plantas. O efeito de doses para número de sementes por vagem foi linear decrescente, apresentando pequena re-

Tabela 3: Desdobramento da interação de métodos de aplicação de molibdênio em relação à utilização de sementes enriquecidas com doses de Mo, para \% de proteína em sementes de soja

\begin{tabular}{lccc}
\hline & \multicolumn{3}{c}{ Sementes enriquecidas com doses de Mo (g ha-1) } \\
\cline { 2 - 4 } Métodos de Aplicação & $\mathbf{0}$ & $\mathbf{4 0 0}$ & $\mathbf{8 0 0}$ \\
\cline { 2 - 4 } & & Proteína em sementes de soja (\%) & 42,60 \\
Sem Aplicação ${ }^{(1)}$ & $39,19 \mathrm{~b}$ & 42,26 & 41,05 \\
Tratamento de Sementes & $42,56 \mathrm{a}$ & 42,41 & 41,22 \\
Via Foliar & $41,68 \mathrm{ab}$ & 40,56 & \\
\hline DMS (5\%) & 2,54 & \\
\hline Médias seguidas de mesma letra, na coluna, não diferem entre si pelo teste de Tukey a 5\% de probabilidade. \\
$\mathrm{y}^{(1)}=0,004263 \mathrm{x}+39,643889 \mathrm{R}^{2}=0,82$
\end{tabular}


dução da quantidade de sementes por vagem. Já para população final de plantas, observou-se diferença para sementes enriquecidas com doses de Mo, ajustando-se a uma equação linear crescente, mostrando que as sementes que foram enriquecidas apresentaram maior taxa de germinação que aquelas para as quais não houve enriquecimento.

Observa-se na Figura 2 que a produtividade de sementes em função de dose foliar de Mo apresentou significância na dosagem de $400 \mathrm{~g} \mathrm{ha}^{-1}$ de Mo, com ajuste a uma equação linear decrescente. Por este fato, o do ajuste decrescente, e pelo fato de a dose de $800 \mathrm{~g} \mathrm{ha}^{-1}$ de Mo não ter sido significativa, pode-se inferir que sementes enriquecidas, com teor de 51,56 $\mathrm{mg} \mathrm{kg}^{-1}$ já teriam a quantidade de Mo suficien- te para obter produtividade desejada $\left(3.00 \mathrm{~kg} \mathrm{ha}^{-1}\right)$, não sendo necessário o uso de Mo por via foliar.

Quanto ao teor de proteína das sementes (Figura 3), observa-se que somente as sementes sem enriquecimento apresentaram diferença, ajustando-se a uma equação linear crescente, ou seja, as plantas advindas de sementes não enriquecidas, ao receberem doses crescentes de Mo foliar, apresentaram aumento do teor de proteína nas sementes, em que a dose foliar de $100 \mathrm{~g} \mathrm{ha}^{-1}$ de Mo apresentou teor de $9 \%$ a mais de proteína do que quando não se aplicou. Não houve diferença entre épocas de aplicação de Mo para teor de proteína, sendo semelhante ao obtido por Moraes et al., (2008), quando se aplicaram Co e Mo em semeadura, em $V_{4}, V_{6}, R_{1}$ e $R_{3}$.

Tabela 4: Valores de F e médias de altura de plantas (AP), número de vagens por planta (VP), número de sementes por vagem (SV), estande (E), massa de 1000 sementes (MM), produtividade (PROD) e percentagem de proteína (PB), de sementes de soja, em função de épocas de aplicação de molibdênio, doses de molibdênio foliar e utilização de sementes enriquecidas com molibdênio

\begin{tabular}{|c|c|c|c|c|c|c|c|}
\hline \multirow[t]{2}{*}{ Tratamentos } & \multirow{2}{*}{$\begin{array}{l}\mathrm{AP} \\
\mathrm{cm}\end{array}$} & $\mathbf{V P}^{(\mathbf{1})}$ & SV & \multirow{2}{*}{$\frac{E}{\text { plantas } \mathbf{h a}^{-1}}$} & \multirow{2}{*}{$\begin{array}{c}\mathrm{MM} \\
\mathbf{g}\end{array}$} & \multirow{2}{*}{$\begin{array}{l}\text { PROD } \\
\text { kg ha }^{-1}\end{array}$} & \multirow{2}{*}{$\begin{array}{c}\text { PB } \\
\%\end{array}$} \\
\hline & & \multicolumn{2}{|c|}{$\mathbf{n}^{\mathbf{0}}$} & & & & \\
\hline \multicolumn{8}{|c|}{ Épocas de aplicação (E) } \\
\hline $\mathrm{V} 4$ & 76,0 & 68,7 & 2,06 & 276.883 & 150,0 & 5.594 & 41,33 \\
\hline Pré-Florescimento & 76,0 & 64,7 & 2,05 & 287.367 & 146,9 & 5.510 & 41,58 \\
\hline R3 & 76,0 & 68,9 & 2,00 & 285.489 & 146,6 & 5.223 & 42,06 \\
\hline R5 & 76,0 & 63,8 & 2,06 & 263.322 & 147,2 & 5.454 & 41,34 \\
\hline \multicolumn{8}{|c|}{ Doses de Mo foliar (D) } \\
\hline \multicolumn{8}{|l|}{$\left(\mathrm{g} \mathrm{ha}^{-1}\right)$} \\
\hline 0 & $68,2^{(2)}$ & 58,4 & $2,10^{(3)}$ & 279.133 & 145,1 & 5.701 & 41,35 \\
\hline 25 & 77,5 & 60,9 & 2,02 & 286.133 & 149,2 & 5.559 & 41,67 \\
\hline 50 & 76,4 & 58,6 & 2,05 & 289.833 & 146,7 & 5.080 & 41,23 \\
\hline 100 & 81,1 & 58,2 & 1,99 & 287.961 & 149,7 & 5.371 & 42,05 \\
\hline
\end{tabular}

Sementes enriquecidas com doses de Mo (S)

\begin{tabular}{|c|c|c|c|c|c|c|c|}
\hline$\left(\mathrm{g} \mathrm{ha}^{-1}\right)$ & & & & & & & \\
\hline 0 & 75,4 & 65,0 & 2,05 & $267.767^{(4)}$ & 146,9 & 5.873 & 40,99 \\
\hline 400 & 75,5 & 64,4 & 2,04 & 279.583 & 147,9 & 5.981 & 41,49 \\
\hline 800 & 76,6 & 62,6 & 2,03 & 287.446 & 148,2 & 5.348 & 42,25 \\
\hline \multicolumn{8}{|l|}{ Teste F } \\
\hline (E) & $0,01^{\mathrm{NS}}$ & $2,18^{\mathrm{NS}}$ & $1,62^{\mathrm{NS}}$ & $2,27^{\mathrm{NS}}$ & $1,82^{\mathrm{NS}}$ & 2,03 NS & $1,02^{\mathrm{NS}}$ \\
\hline (D) & $8,59^{* * *}$ & $9,34^{\mathrm{NS}}$ & $4,05^{* *}$ & $1,19^{\mathrm{NS}}$ & $3,43^{\mathrm{NS}}$ & $1,73^{\mathrm{NS}}$ & $1,17^{\mathrm{NS}}$ \\
\hline (S) & $0,16^{\mathrm{NS}}$ & $0,20^{\mathrm{NS}}$ & $0,24^{\mathrm{NS}}$ & $3,48^{*}$ & $0,49^{\mathrm{NS}}$ & $1,40^{\mathrm{NS}}$ & $4,70^{*}$ \\
\hline (E)x(D) & $0,40^{\mathrm{NS}}$ & $0,53^{\mathrm{NS}}$ & $1,12^{\mathrm{NS}}$ & $2,25^{\mathrm{NS}}$ & $0,78^{\mathrm{NS}}$ & $0,84^{\mathrm{NS}}$ & $0,75^{\mathrm{NS}}$ \\
\hline (E)x(S) & $0,13^{\mathrm{NS}}$ & $0,35^{\mathrm{NS}}$ & $0,90^{\mathrm{NS}}$ & $1,99^{\mathrm{NS}}$ & $0,71^{\mathrm{NS}}$ & $1,15^{\mathrm{NS}}$ & $0,42^{\mathrm{NS}}$ \\
\hline (D) $x(S)$ & $0,37^{\mathrm{NS}}$ & $0,74^{\mathrm{NS}}$ & $1,13^{\mathrm{NS}}$ & $0,89^{\mathrm{NS}}$ & $0,64^{\mathrm{NS}}$ & $2,30^{*}$ & $3,98^{* *}$ \\
\hline$(\mathrm{E}) \mathrm{x}(\mathrm{D}) \mathrm{x}(\mathrm{S})$ & $0,15^{\mathrm{NS}}$ & $0,68^{\mathrm{NS}}$ & $0,61^{\mathrm{NS}}$ & $1,11^{\mathrm{NS}}$ & $0,64^{\mathrm{NS}}$ & $0,84^{\mathrm{NS}}$ & $0,31^{\mathrm{NS}}$ \\
\hline DMS (5\%) & 6,85 & 11,10 & 0,08 & 26876 & 4,20 & 1365,7 & 1,25 \\
\hline C.V. $(\%)$ & 14,66 & 16,04 & 7,63 & 15,67 & 7,78 & 38,41 & 4,87 \\
\hline Média Geral & 75,8 & 64,0 & 2,04 & 278.265 & 147,7 & 5.767 & 41,58 \\
\hline
\end{tabular}

(1) Dados transformados em $(\mathrm{X}+0,5)^{\wedge} 0,5$

Médias seguidas de mesma letra, na coluna, não diferem entre si pelo teste de Tukey a 5\% de probabilidade.

Ns $=$ não significativo; $*$ = significativo a $5 \%$ de probabilidade; $* *=$ significativo a $1 \%$ de probabilidade.

$\mathrm{y}^{(2)}=0,109293 \mathrm{x}+71,02156 \mathrm{R}^{2}=0,74 ; \mathrm{y}^{(3)}=-0,000888 \mathrm{x}+2,07821 \mathrm{R}^{2}=0,70$;

$\mathrm{y}^{(4)}=-14,6861 \mathrm{x}^{2}+1845,385 \mathrm{x}+244254,091 \mathrm{R}^{2}=0,97$

Rev. Ceres, Viçosa, v. 64, n.3, p. 282-290, mai/jun, 2017 
Segundo Mengel \& Kirkby (2001), o Mo faz parte da enzima redutase de nitrato, que catalisa a redução de $\mathrm{NO}_{3}^{-}$ a $\mathrm{NO}_{2}$ - . Além desta participação, o molibdênio também faz parte da molécula da nitrogenase, que catalisa a redução do $\mathrm{N}_{2}$ atmosférico a $\mathrm{NH}_{3}$. Espera-se, por isso, que quanto maior a quantidade de Mo presente nas plantas, maiores os valores de $\mathrm{N}$ na planta.
Podem-se verificar os dados sobre o aumento de proteína na Figura 3, sendo que o modelo de ajuste das equações foi linear crescente. $\mathrm{O} \mathrm{N}$ faz parte da constituição das proteínas; sendo assim, é esperado que um tratamento que aumente o teor desse nutriente influencie diretamente o teor de proteína nas sementes.

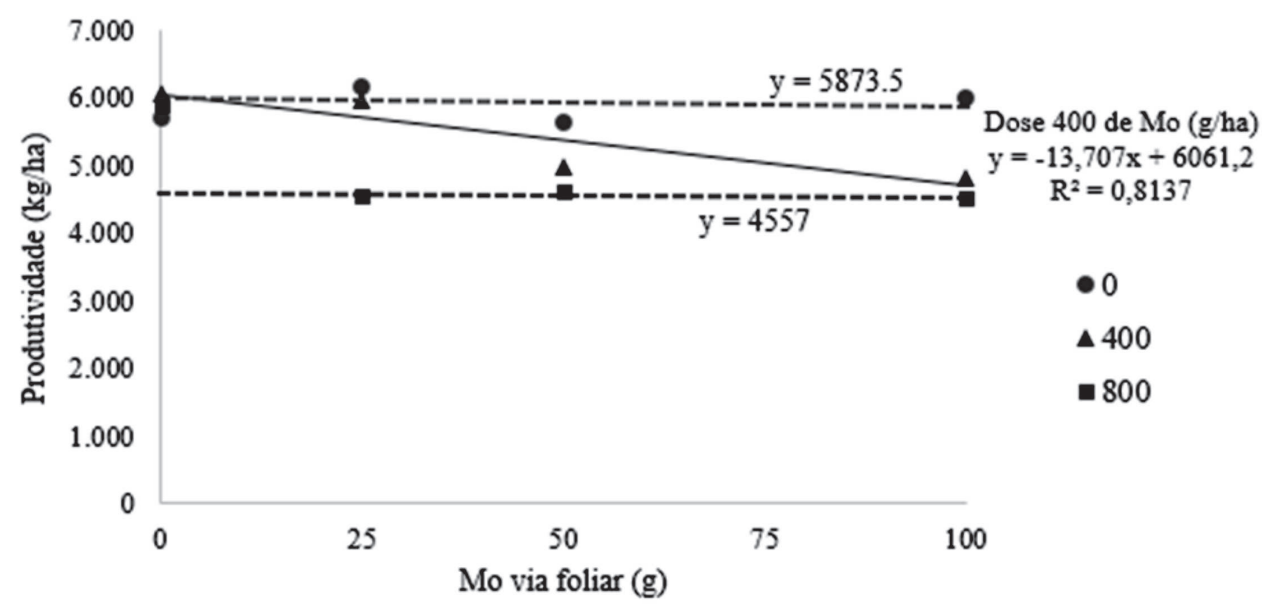

Figura 2: Desdobramento da interação entre doses de Mo foliar e sementes enriquecidas, para produtividade $\left(\mathrm{kg} \mathrm{ha}^{-1}\right)$.

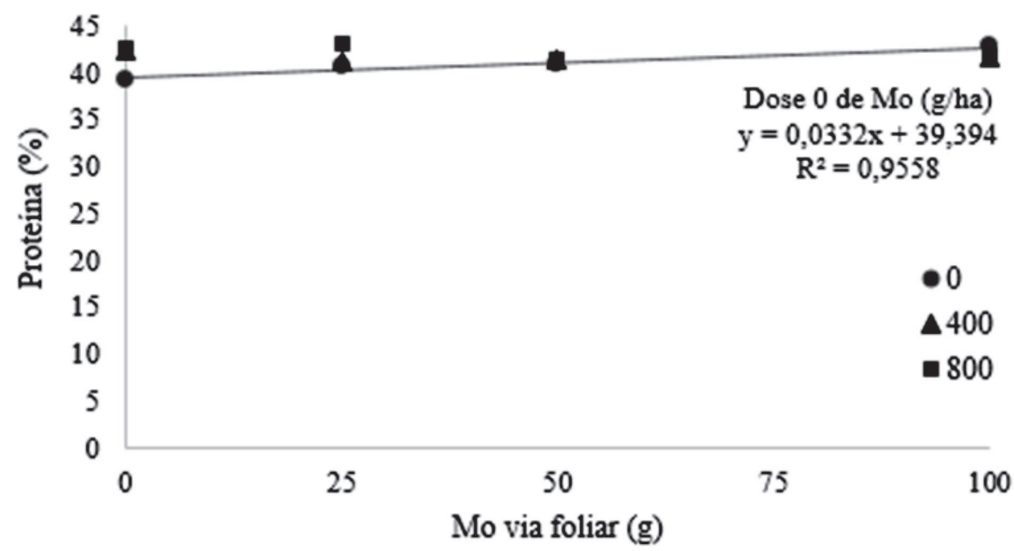

Figura 3: Desdobramento da interação de doses de Mo foliar, em relação às sementes enriquecidas com doses de Mo, para \% de proteína.

\section{CONCLUSÕES}

O teor de proteína das sementes é $9 \%$ maior com a presença de Mo, independentemente do modo como esse é fornecido.

A utilização de Mo influi positivamente na produtividade de soja, quando fornecido pelo uso de sementes enriquecidas com teor de até $51,56 \mathrm{mg} \mathrm{kg}$, ou por aplicação por via foliar, com doses entre 25 e $50 \mathrm{~g} \mathrm{ha}^{-1}$.

\section{AGRADECIMENTOS}

Os autores agradecem à Fundação de Amparo e Pesquisa do Estado de São Paulo - FAPESP, pela bolsa de estudo.

\section{REFERÊNCIAS}

Albino UB \& Campo RJ (2001) Efeito de fontes e doses de molibdênio na sobrevivência do Bradyrhizobium e na fixação biológica de nitrogênio em soja. Pesquisa Agropecuária Brasileira, 36:527-534

AOSA - Association of Official Seed Analysts (1983) Seed vigour testing handbook. East Lasing, AOSA. 88p.

Brasil (2009) Ministério da Agricultura, Pecuária e Abastecimento. Regras para análise de sementes. Brasília, Mapa/ACS. $395 \mathrm{p}$.

Campo RJ, Albino UB \&Hungria M (1999) Métodos de aplicação de micronutrientes na nodulação e na fixação de $\mathrm{N}_{2}$ em soja. Disponível em: http://www.infoteca.cnptia.embrapa.br/ bitstream/doc/461380/1/pesqAnd019.pdf Acessado em: 25 de janeiro de 2015.

Rev. Ceres, Viçosa, v. 64, n.3, p. 282-290, mai/jun, 2017 
Campo RJ \& Hungria M (2003) Enriquecimento de sementes de soja com molibdênio como fator de aumento da eficiência da fixação biológica do nitrogênio e do rendimento da soja. In: Reunião de pesquisa de soja da região central do Brasil, Uberaba. Anais, Embrapa/Epamig. p.156-157.

Cheng T (1985) The effect of the seed treatment with microelements upon the germination and early growth of wheat. Scientia Sinica, 44:129-135.

Conab - Companhia Nacional de Abastecimento (2014) Acompanhamento da safra brasileira de grãos 2013/2014 - nono levantamento - Junho/2014. Disponível em: <http://www.conab. gov.br/OlalaCMS/uploads/arquivos/14_07_16_14_59_31_boletim_graos_junho_2014.pdf>. Acessado em: 25 de janeiro de 2015 .

Embrapa - Empresa Brasileira de Pesquisa Agropecuária (2003) Tecnologias de produção de soja - Região Central do Brasil 2004. Londrina, Embrapa/Epamig. 212p. (Sistemas de Produção, 4).

Fehr WR, Caviness CE, Burmood DT \& Pennington JS (1971) Stage of development descriptions for soybeans, Glycine max (L.) Merrill. Crop Science, 11:929-931.

Ferreira DF (2010) SISVAR. Sistema de análise de variância. Versão 5.3. Lavras, UFLA. CD ROM.

Harris HB, Parker MB \& Johnson BJ (1965) Influence of molybdenum content in soybean seed and other factors associated with seed source on progeny response to applied molybdenum. Agronomy Journal, 57:397-399.

Lima TC (2009) Enriquecimento de sementes de feijão com Molibdênio e seus efeitos na qualidade fisiológica de Sementes e na produtividade em três gerações de Plantio. Dissertação de Mestrado. Universidade Federal de Viçosa, Viçosa. 52p.

Malavolta E (2006) Manual de nutrição mineral de plantas. São Paulo, Agronômica Ceres. 638p.

Malavolta E, Vitti GC \& Oliveira AS (1997) Avaliação do estado nutricional das plantas: princípios e aplicações. $2^{\mathrm{a}}$ ed. Piracicaba, Potafos. 319p

Mengel K \& Kirkby EA (2001) Principles of plant nutrition. 5 ed. Dordrecht, Kluwer Academic Publishers. 849p.
Meschede DK, Braccini AL, Braccini MCL, Scapim CA \& Schuab SRP (2004) Rendimento, teor de proteínas nas sementes e características agronômicas das plantas de soja em resposta à adubação foliar e ao tratamento de sementes com Molibdênio e Cobalto. Acta Scientiarum, 26:139-145.

Moraes LMF, Lana RMQ, Mendes C, Mendes E, Monteiro A \& Alves JF (2008) Redistribuição de molibdênio aplicado via foliar em diferentes épocas na cultura da soja. Ciência e Agrotecnologia, 32:1496-1502.

Nakao AH, Vazquez GH, Oliveira CO, Silva JC \& Souza MFP (2014) Aplicação foliar de molibdênio em soja: efeitos na produtividade e qualidade fisiológica da semente. Enciclopédia Biosfera, 10:343-352.

Oz M (2008) Nitrogen rate and plant population effects on yield and yield componentes in soybean. African Journal of Biotechnology, 7:4464-4470.

Parducci S, Santos OS, Camargo RP, Leão RMA \& Batista RB (1989) Micronutrientes Biocrop. Campinas, Microquímica. $101 \mathrm{p}$.

Pessoa ACS (1998) Atividades de nitrogenase e redutase do nitrato e produtividade do feijoeiro em resposta à adubação com molibdênio e fósforo. Tese de Doutorado. Universidade Federal de Viçosa, Viçosa. 151p.

Possenti JC \& Villela FA (2010) Efeito do molibdênio aplicado via foliar e via sementes sobre o potencial fisiológico e produtividade de sementes de soja. Revista Brasileira de Sementes, 32:143150 .

Possenti JC, Villela FA \& Delqui EM (2009) Componentes de Rendimento de Soja (Glycine max) produzida com o uso de sementes enriquecidas por molibdênio. In: $1^{a}$ Reunião Paranaense de Ciência do Solo, Pato Branco. Anais, Universidade Tecnológica Federal do Paraná. p.110-104.

Santos MJ (2012) Extração e determinação de Mo em três argissolos do nordeste cultivados com cana-de-açúcar. Dissertação de mestrado. Universidade Federal Rural de Pernambuco, Recife. 66p.

Souza CA, Gava F, Casa RT, Bolzan JM \& Kuhnem Junior PR (2010) Relação entre densidade de plantas e genótipos de soja Roundup Ready ${ }^{\mathrm{TM}}$. Planta Daninha, 28:887-896. 